\section{Bacteriological Notes}

Microscopical.-On three separate occasions, the first on the fourteenth day, the urine was examined for spirochaetes, and these were found. The specimens were centrifuged, and the deposit examined under dark-ground illumination, and also stained by Fontana's silver method. It was found necessary to obtain a very fresh specimen in order to observe the movements, which consisted of rotation and undulation. In stained preparations the spirochaetes were not very numerous, but about a dozen or so of these were seen, and one (the first) preparation was photographed. There was a distinct hook-like tendency at the termination of the spirochaete, but the textbook well-marked hooking was only obvious in a few specimens. It must be remembered that the urine has an action on the morphological appearances, and tends to make the organism appear somewhat atypical.

Biological.-Rather late in the disease animal inoculation of the centrifuged deposit was tried, and a guinea-pig was injected intraperitoneally. No effects were observed, the animal being killed a fortnight after injection.

A. D. Gardner ${ }^{1}$ states that the organism occurs both in fresh and in sea water, and can be cultivated therefrom.

Dr. R. A. O'Brien very kindly consented to test to serological agglutination against the leptospira with a specimen of the patient's serum, but suitable strains are not for the moment available.

REFERENCE

${ }^{1}$ Gardner, A. D.: Bacteriology for Medical Students and Practitioners, 1933, p. 195.

\section{PULMONARY TUBERCULOSIS AFTER SUNBATHS}

BY

A. HOPE GOSSE, M.D.Cantab., F.R.C.P.Lond.

PHYSICIAN TO THE BROMPTON HOSPITAL FOR DISEASES OF THE CHEST AND

\section{G. S. ERWIN, M.B., CH.B. N.Z.}

ASSISTANT RESIDENT MEDICAL OFFICER, BROMPTON HOSPITAL

The decline in the annual death rate from tuberculosis of the lungs has been so steady since 1860, apart from the war years, that this disease may reasonably be expected to be rare in twenty or thirty years. While therapeutics have helped, the main cause of this magnificent improvement is to be found in the amelioration of social conditions and the education of the patients and the public in preventive measures. The relation of any change in social customs to the development of tuberculosis should be carefully watched. Towards the end of the hot summer of 1933 there appeared in our ward: patients with recently developed pulmonary tuberculosis, who were heavily tanned from sunbathing. We therefore decided to make a clinical study of the relation, if any, between this hobby of sunbathers and this disease of the lungs, and we here present the results and some conclusions.

Careful inquiry in each case elicited the fact that out of sixty-six cases of pulmonary tuberculosis admitted under our care between August and December, 1933, the onset, or exacerbation, of symptoms in eleven cases followed sunbathing. Brief histories of these cases are appended, and a study of them shows that there are certain points of similarity in the development of symptoms.

\section{Case Notes}

Case 1.-A male, aged 33, admitted August 30th, 1933. In spite of night sweats in January for two weeks, he sunbathed in July. Three weeks later he noticed a cough and felt weak. Sputum positive, $x$ ray positive, on admission.

Case 2.-A male, aged 19, admitted September 13th, 1933. Had haemoptysis in April, 1933. In the following June he sunbathed for one whole day; felt dizzy and lost appetite for two weeks. Sputum positive, $x$ ray positive, $\mathrm{cn}$ admissi $n$.

Case 3.-A man, aged 29, admitted September 20th, 1933. Pneumonia last year; no residual cough. Cough commenced in August, 1933, with one haemoptysis. Sputum positive. In spite of this he sunbathed at Margate for a week, but felt dizzy and sweated at night. Sputum positive, $x$ ray positive, on admission.

Case 4.-A female, aged 20, admitted September 20th, 1933. Quite well and sunbathed during week-ends until, on August Bank Holiday, after sunbathing at Folkestone, felt short of breath and ill for the next few days. Haemoptysis ten days later, then cough developed. Sputum none, $x$ ray positive, on admission.

Case 5.-A female, aged 25, admitted September 2sth, 1933. Influenza three previous winters. Quite well till she sunbathed at Broadstairs for one week in June, and on one day had a "bilious" attack, which was attributed to excess of sunbathing. Six weeks later developed a cough. Sputum positive, $x$ ray positive, on admission.

Case 6.-A female, aged 19, admitted November 1st, 1933. Pneumonia in January, 1933. Quite well when she sunbathed at Margate for a week in July, 1933, and felt no ill effects. In September and October dyspnoea. Sputum negative, $x$ ray positive, on admission.

Case 7.-A male, aged 20, admitted November 3rd, 1933, Quite well while he extensively sunbathed at his home and for a week at Westcliff in August, 1933. Haemoptysis in October, 1933, first symptom. Sputum negative, $\boldsymbol{x}$ ray positive, on admission.

Case 8.-A woman, aged 26, admitted November 13th, 1933. In spite of a cough sunbathed for one week early in September, 1933. Did not feel well all the week. Five weeks later, blood-stained sputum. Sputum positive, $x$ ray positive, on admission.

Case 9.-A female, aged 20, admitted November 22nd, 1933. Dry pleurisy 1932 ; cough persisted. Sunbathed on the river during the week-ends all through the summer of 1933. Haemoptysis in November, 1933. Sputum positive, $x$ ray positive, on admission.

Case 10.-A female, aged 23, admitted December 13th, 1933. Cough since August, 1933. Sunbathed in August for one week. No ill effects at the time. Sputum positive, $x$ ray positive, on admission.

Case 11.-A male, aged 24, admitted December 30th, 1933. Slight cough last winter. Quite well in July, 1933, when he sunbathed for a quarter of an hour each day. No ill effects noticed. Had haemoptysis in November, 1933. Sputum positive, $x$ ray positive, on admission.

The age incidence ranged between 19 and 33, years common to sunbathing and to the production of the exudative type of the disease, which was the one present in all these eleven cases on radiological appearances. On the other hand, although there was a considerable proportion of the fibroid type of disease among the other fifty-five patients, each one of them denied indulging in sunbathing during the summer, and an absence of sun pigmentation supported their statements as far as it could. The fibroid type was present in some patients whose age was greater than the average of sunbathers, and in others who had passed the exudative stage. Many of these patients had a long history of tuberculosis, and had been deliberately warned by their doctors against the danger of sunbathing.

That the abnormal exposure of the usually covered skin surfaces to the action of the sun's rays aggravates the development of pulmonary tuberculosis is a conclusion suggested by these eleven cases and others we 
have examined, and we believe this new social custom has elements of danger if indulged in extensively and indiscriminately. This is, of course, in accordance with the known danger of treating apyrexial cases of pulmonary tuberculosis by insolation or carefully graded exposure of the skin to the sun. Years ago it was found by one of us, as by many others who tried it as a therapeutic procedure, that pyrexia and increased activity of the disease sometimes resulted. The danger of artificial sunlight to a patient with tuberculosis of the lungs must also be remembered.

The exact manner in which sunbathing may aggravate tuberculosis is not understood. We do not know whether long sunbaths raise the body temperature in healthy young adults or possibly only in those in a hyperallergic state. For this latter possibility there is some support in the fact that in our cases several patients showed an immediate reaction in the form of malaise and sweats, as well as a delayed reaction some weeks to three or four months later in the more dramatic form of haemoptysis among other symptoms.

A further point of interest is the fact that our clinical study automatically terminated it:elf at the end of the year by the non-appearance of new cases with a relevant history of sunbathing. There were none in January or February, 1934. Hence we conclude that the ill effects of sunbathing may be expected to manifest themselves within four or five months of exposure.

\section{Conclusions}

In view of our opinion from the experiences gained from this clinical study we venture to draw the following conclusions :

1. It is dangerous for anyone who has had haemoptysis, especially if recent, to sunbathe until tuberculosis of the lungs has been excluded by a thorough examination, including an $x$-ray examination of the chest.

2. It is inadvisable for people who have recently lost weight, or who are feeling abnormally tired, or who have other suspicious symptoms to sunbathe without the same precautions.

3. Sunbathers who feel tired or feverish, or who perspire at night after a sunbath, should take their temperature, and, if it is above $99^{\circ} \mathrm{F}$. in the evening, no more sunbaths should be attempted until they have been passed as fit.

At the June meeting of the Central Midwives Board for England and Wales a letter was read stating that the Minister of Health had approved the existing Rules for a further period of three months from June 30th. A letter was received from the Women Public Health Officers' Association suggesting that, in view of the difficulties experienced by health visitors whose names had been removed from the Roll under Section 3 (2) of the Midwives Act; 1926, in undergoing further training before the restoration of their names, the Board might restore the names on an undertaking being given by the health visitors concerned that they would not practise midwifery without undergoing such further courses of training as the Board deemed necessary. It was agreed to reply that women whose names have been removed from the Roll under Section 3 (2) and who are in actual practice as health visitors be re-enrolled on application and the payment of the restoration fee, and that this instruction should apply to women in practice as health visitors whose names have been removed from the Roll and who would have been ordered further training as a condition precedent to the restoration of their names.

\section{Clinical Memoranda}

\section{DRY PLEURISY WITH HIGH EOSINOPHIL LEUCOCYTOSIS}

The following is a case of dry pleurisy with a high degree of eosinophilia.

An Indian Christian, aged 21, was admitted to hospital on January 21st, 1933, complaining of cough, low fever, and pain in the chest for one month. He had had cholera in May and heatstroke in June, 1932, but no other previous illness. His trouble had started with a slight attack of cold, passing on to severe spasms of cough, which lasted for half an hour (r $r$ so and caused breathlessness. At first there was no expectoration; later a thin, white, and slightly frothy sputum was brought up. The fever had reached $100^{\circ}$ or $101^{\circ} \mathrm{F}$., and was often preceded by rigor. Occasionally he passed into a semiconscious state, especially after an attack of coughing, accompanied by profuse perspiration. Insomnia was marked.

Physical examination showed, in a thinly built, rather toxic-looking youth, signs of disease at the right pulmonary base-namely, a dull percussion note, diminution of vocal fremitus and resonance and of breath sounds, fine intrapulmonary crepitations, and a loud pleural rub. Elsewhere there were no abnormal signs. Radiological examination revealed infiltration and pleural shadow of the lower part of the right lung; no indication of involvement of the liver could be made out, and the right diaphragm moved fairly even when later there was some swelling of the hepatic region, and the possibility of liver abscess was considered.

The patient's clinical condition varied very little during his three months' stay in hospital, except that the fever became very slight. Pain was most acute, and insomnia very troublesome. He developed dry pleurisy on the left side also, but the signs, radiological and clinical, on both sides gradually cleared up. Needling revealed no fluid, nor was an attempt to relieve the pleural pain, by the induction of artificial pneumothorax, successful. He was given a course of emetine injections on account of the swelling over the liver, and a clinical effect was certainly produced.

So far the probable diagnosis would have been tuberculosis, or possibly amoebiasis, but the pathological examinations introduced a disturbing factor. The sputum was consistently negative for tubercle bacilli and fungi, the blood showed no parasites, and the stools contained no pathogenic organisms. The leucocyte count, however, was abnormal; successive counts were as follows:

\begin{tabular}{|c|c|c|}
\hline Dat & & \\
\hline January & $23 \mathrm{rd}$ & 1933 \\
\hline & $\begin{array}{l}27 \text { th, } \\
\text { 30th, }\end{array}$ & ", \\
\hline February & 2nd, & $"$ \\
\hline$"$ & $\begin{array}{l}\text { 9th, } \\
\text { 14th, }\end{array}$ & $"$ \\
\hline & 25th, & $\because$ \\
\hline March & $\begin{array}{l}\text { 6th, } \\
\text { 23rdd, }\end{array}$ & ," \\
\hline
\end{tabular}

\begin{tabular}{ll} 
& \multicolumn{2}{c}{ Tota } \\
& \multicolumn{2}{c}{ blo } \\
$\ldots$ & $\cdots$ \\
$\ldots$ & $\cdots$ \\
$\cdots$ & $\cdots$ \\
$\cdots$ & $\cdots$ \\
$\cdots$ & $\cdots$ \\
$\cdots$ & $\cdots$ \\
$\cdots$ & $\cdots$ \\
$\cdots$ & $\cdots$ \\
$\ldots$ & $\cdots$
\end{tabular}

Eosinophils per cent. 80
78 78
82 79
81 81 81
82 77

The eosinophils were both $\mathrm{bi}$ - and tri-lobed. The treatment was mainly symptomatic, but, in addition to the emetine, on the possibility of a fungus infection of the lung, large doses of iodide were given for a time, and, on the theory of some allergic condition, auto-blood injections.

Being tired of hospital, the patient was removed by his relations considerably improved, and various indigenous treatments were trieci until, in May, he came under the care of the junior author. He was then experiencing every afterncon very severe attacks of cough, followed by thin, frothy sputum, often tinged with blood. After these attacks he passed into a hysterical or semi-comatose condition lasting an hour or two. He was given one dose of $1 / 2$ grain ephedrine hydrochloride, which was followed by an alarming condition of collapse, with a feeble pulse of 140 per minute; from this he recovered after volatile stimulants, bandaging the lower limbs, and raising the foot of the bed. From the next day he was given ephedrine in doses of 1/12 grain four-hourly, and a mixture containing iodide, bromide, and tincture of belladonna. Whenever he experienced a choking sensation he was given a dose of "syrup sourkalp" (a preparation of Ephedra vulgaris). He was at the same time takıng various Ayurvedic 